Jean-Paul GaUdiLlière, Hugues JALlon, (dirs), « De quoi avons-nous (vraiment) besoin ? Bonheur, consommation, capitalisme ", Mouvements des idées et des luttes, 54 .

Paris, Éd. La Découverte, 2008, 144 p.

Chadi El Nar

\title{
OpenEdition
}

Journals

Édition électronique

URL : http://journals.openedition.org/questionsdecommunication/198

DOI : 10.4000/questionsdecommunication.198

ISSN : 2259-8901

Éditeur

Presses universitaires de Lorraine

Édition imprimée

Date de publication : 1 décembre 2009

ISBN : 978-2-8143-0003-3

ISSN : 1633-5961

Référence électronique

Chadi El Nar, « Jean-Paul gaudilıı̇erre, Hugues Jallon, (dirs), « De quoi avons-nous (vraiment) besoin? Bonheur, consommation, capitalisme », Mouvements des idées et des luttes, 54. », Questions de

communication [En ligne], 16 | 2009, mis en ligne le 19 janvier 2012, consulté le 21 décembre 2020.

URL : http://journals.openedition.org/questionsdecommunication/198; DOI : https://doi.org/10.4000/ questionsdecommunication.198

Ce document a été généré automatiquement le 21 décembre 2020.

Tous droits réservés 


\section{Jean-Paul GAUDILLIÈRE, Hugues JALLON, (dirs), « De quoi avons-nous (vraiment) besoin ? Bonheur, consommation, capitalisme ", Mouvements des idées et des luttes, 54.}

Paris, Éd. La Découverte, 2008, 144 p.

Chadi El Nar

\section{RÉFÉRENCE}

Jean-Paul GAUDILLIÈRE, Hugues JALLon, (dirs), « De quoi avons-nous (vraiment) besoin? Bonheur, consommation, capitalisme », Mouvements des idées et des luttes, 54., Paris, Éd. La Découverte, 2008, 144 p.

1 De toute évidence, la $54^{\mathrm{e}}$ livraison de la revue Mouvements des idées et des luttes est au cœur du débat social, politique et intellectuel, en abordant plusieurs thèmes phares comme le besoin, le bonheur, la consommation, le capitalisme, l'écologie, etc. Dans ce dossier divisé en trois parties(«Besoin et aliénation»; «Besoins et bonheur»; «Besoins, économie et développement »), les auteurs cherchent à " penser les besoins » en distinguant les «faux » des "vrais», ceux qui sont " authentiques » ou "factices", et en répondant à plusieurs interrogations telles: "Quels critères déterminent la valeur des besoins humains? Imagine-t-on un État démocratique qui se chargerait de déterminer (même avec l'aide de citoyens investis dans des instances de démocraties participatives) les "vrais" besoins de ses citoyens? » (p.8).

2 Jean-Paul Gaudillière et Hugues Jallon précisent que « l'enjeu de ce numéro n'est pas la liste de ce dont nous aurions (vraiment) besoin mais la façon dont on peut repenser le problème, revisiter- du point de vue des besoins- des catégories comme celles de 
progrès technique, d'abondance, de bonheur ou encore d'aliénation»(p.9). Pour atteindre cet objectif, de nombreux sujets sont traités : l'aliénation, la réalisation de soi, l'économie de bonheur, les techniques et l'augmentation de la productivité, la conscience écologique, la fabrication des besoins, les nouvelles figures du capitalisme, la révolution numérique, etc. Ce sont les travaux de Theodor W. Adorno et l'état des lieux d'Agnès Heller qui fondent la réflexion engagée dans l'ouvrage. Pour le premier, et à partir d'un texte écrit lors de l'exil américain du chercheur, la théorie des besoins est socialement marquée. Elle pose la question de la satisfaction sans différenciation préalable entre les bons et les mauvais besoins, authentiques et artificiels, vrais et faux (p.15). A contrario, Agnès Heller illustre- « dans le contexte des discussions des années 1970 sur l'autogestion et les nouveaux mouvements sociaux » (p.13)- la différence entre " vrais » et « faux » sous trois angles : elle rejette la division entre besoins « réels » et " imaginaires », accepte celle entre «bons » et "mauvais » dans un sens concret, et adopte la distinction entre besoins "préférés » et "non préférés", à condition toutefois que celle-ci soit associée aux valeurs.

3 À ce sujet, Alain Deneault traite de la valeur monétaire en s'appuyant sur les travaux de Georg Simmel La philosophie de l'argent (trad. de l'allemand par Sabine Cornille et Philippe Ivernel, Paris, Presses universitaires de France, [1907] 1987- p. 34), pour qui l'argent est un méta-moyen permettant l'accès aux choses. Alain Deneault passe d'une description utopique de la valeur monétaire à un discours polémique en indiquant que l'argent provoque une série de pathologies qui vont du fait d'être blasé au cynisme, en passant par l'avarice et la cupidité (p.39). Dans le prolongement, Stéphane Haber critique le capitalisme et le lien qu'il entretient avec la dépossession. Il définit l'«aliénation » et pose l'idée selon laquelle la critique de celle-ci ne se limite pas aux modèles qui composent les systèmes aliénants tels l'argent, le capital (au sens de Marx) ou l'État bureaucratique. En effet, historiquement, philosophie de l'aliénation et critique pré-écologique de la modernité capitaliste sont fortement articulées. Aussi conclut-il qu' « aujourd'hui, ce sont les thèmes et les combats de l'alter-mondialisme qui constituent les incitations les plus stimulantes à redécouvrir la fécondité du vieux concept marxien d'aliénation » (p.53).

4 C'est la question du "système technique et déshumanisation " qui intéresse Thierry Paquot. Citant Günther Anders (L'obsolescence de l'homme. Sur l'âme à l'époque de la deuxième révolution industrielle, trad. de l'allemand par Christophe David, Paris, Éd. de L'Encyclopédie des nuisances/, Éd. Ivréa [1956] 2002; voir aussi Daglind Sonolet, Günther Anders: phénoménologue de la technique, Pessac, Presses universitaires de Bordeaux, 2006) et Bernard Charbonneau (voir notamment Le jardin de Babylone, Paris, Gallimard, 1969, nouvelle édition, Paris, Éd. de l'Encyclopédie des nuisances, 2002 ; Christian Roy, "Aux sources de l'écologie politique: le personnalisme "gascon" de Bernard Charbonneau et Jacques Ellul », Canadian Journal of History/Annales canadiennes d'histoire, XXVII, 1992, pp.67-100), il considère que le déploiement des techniques détruit l'humanité qui se retrouve "sans monde». Ainsi Bernard Charbonneau considère-t-il que le système technique tente le progrès pour le progrès, ce qui engendre et facilite l'homogénéisation des humains. Mais Thierry Paquot estime que ce sont les loisirs qui sont évoqués par les études et non la consommation. Cette dernière s'impose progressivement sous la pression des industriels du loisir qui occupent le temps libre. Selon le chercheur, que ce soit à travers le désir ou les besoins, le bonheur 
est fondé sur l'accumulation: "Cette conception du bonheur par la consommation légitime le système productif et ses innovations techniques » (p.64).

Élise Requilé poursuit en traitant du «développement personnel aujourd'hui » qui, d'après elle, symbolise un secteur de l'édition et des services, une démarche et l'ensemble des pratiques sur lesquelles elle s'appuie. "Le développement personnel » s'inscrit donc davantage dans une démarche de bien- ou de mieux-être- que dans une démarche de soin approfondie. Ces pratiques s'appuient sur l'idée d'une sousexploitation du potentiel humain, qui englobe les capacités essentielles de l'individu » (p.68). Elle cite également les « principes et notions clefs du développement personnel » en précisant que ceux-ci sont « la philosophie » implicite qui mesure le rapport à soi et au monde que véhicule le développement personnel. Elle évoque aussi les «trois pôles d'influence: psychotechniques, approche humaniste et thérapies cognitivocomportementales ", et la " gestion des effets, au détriment de la prise en compte des causes » en soulignant que "le développement personnel s'appui [...] sur un ensemble d'approches qui visent le traitement et la gestion des effets en laissant de côté le travail d'explication des causes du malaise psychique ou physique et des conditions de leur production » (p.73).

Quant à Ilana Löwy, elle s'intéresse au point de vue anglo-saxon de l'économie de l'argent car c'est aux États-Unis et en Grande-Bretagne que les recherches sur le bonheur se sont initialement développées. La chercheuse traite du lien entre action politique et bonheur et évoque les chercheurs britanniques: "Le New Labour et la science du bonheur ", "Richard Layard et la qualification du bonheur ", "Richard Sennet et le bonheur des artisans ", « Richard Wilkinson ou les conséquences mortelles du déficit de bonheur». En conclusion, elle évoque "l'économie du bonheur entre realpolitik et utopie " pour en venir à ce constat: "L'écart entre l'aspiration de créer une société égalitaire et fraternelle, et celle de construire une société qui rende possible la recherche de bonheur et protège la propriété privée, résume bien les tensions de la nouvelle "économie de bonheur"« (p.86).

Dans un entretien mené par Vincent Bourdeau, Fabrice Flipo et Julien Vincent, Gareth Stedman Jones étudie la notion de besoins collectifs. Il considère que les besoins sont toujours individuels: "Je ne crois pas que la notion de besoins collectifs soit très éclairante » (p.88). D'après lui, cette question soulève le problème des relations entre les personnes et les choses, et suggère un état de la réalité qui répond aux besoins individuels. En outre, dans « Écologie en temps de guerre », Mike Davis explique que les Américains connus par leur forte consommation, sont devenus écologistes pendant la Seconde Guerre mondiale en encourageant le covoiturage, en «recyclant les idées et non pas seulement les matériaux " et en incitant les citoyens à n'acheter que le nécessaire. (p.96). Enfin, Jean-Paul Gaudillière analyse la relation entre connaissance, innovations et besoins. Il estime que la société de la connaissance met en débat l'émergence d'un nouveau capitalisme- dit « cognitif » ou " immatériel »- et espère que les discussions sur la révolution numérique aboutiront à des critiques sur la consommation, les besoins et le travail qui, d'après lui, ont été effacées des mémoires au cours des décennies de la «fin des utopies » (p.110). À noter que cette livraison est complétée par un entretien avec Joan Martinez Alier («De l'économie à l'écologie en passant par les Andes ») et par une contribution de Gilbert Achcar («L'orientalisme à rebours : de certaines tendances de l'orientalisme français après $1979 »)$. 
8 Terminons sur un constat révélé dans ces travaux ici rassemblés : «Si la leçon est $a$ priori radicalement opposée à celle proposée par les utopies de la technique ou de la décroissance volontaire, elle les rejoint sur un point essentiel : la défense de l'idée selon laquelle l'égalité, l'investissement collectif dans l'élaboration de biens ou de services "publics" créent du bonheur et satisfont "d'autres besoins"« (p.11).

\section{AUTEURS}

\section{CHADI EL NAR}

CREM, université Paul Verlaine-Metz

chadyelnar@hotmail.com 